ser Arbeit kann angenommen werden, dass diese Variablen negative Gefühlserleben und damit auch die kognitiven Wirkungen gewalthaltiger Spiele auf explizite Gedächtnisleistungen moderieren könnten.

\title{
1.5 Zielsetzung der geplanten Untersuchung
}

\subsubsection{Bewertung der empirischen Datenlage}

In Kapitel 1.2.2 konnte gezeigt werden, dass erhöhte Nutzungszeiten sowie eine Präferenz für Unterhaltungs- oder gewalthaltige Angebote mit schlechteren Schulleistungen in Zusammenhang stehen. Für das Fernsehen liegen hierzu Metaanalysen sowie Längsschnittstudien vor, die zumindest für die letzten zehn Jahre ein stabiles Befundmuster erkennen lassen und inzwischen auch die Annahme einer kausalen Wirkrichtung von der Mediennutzung auf die Schulleistung nahelegen (vgl. Kapitel 1.2.2.1). In weit geringerem Maße bestehen solide Forschungsdaten zu der Frage, wie sich Computerspielnutzung auf schulische Leistungen auswirkt (vgl. Kapitel 1.2.2.2). Die vorhandenen Querschnittstudien weisen zwar ebenfalls auf einen negativen Zusammenhang hin, der insbesondere bei der Nutzung gewalthaltiger Spiele beobachtet werden kann. Eine kausale Wirkrichtung vom Computerspielen auf schlechtere Schulleistung kann jedoch aus den Studien aufgrund eines Mangels an längsschnittlichen und experimentellen Wirkungsstudien bislang nicht in eindeutiger Weise abgeleitet werden, auch wenn in einigen Querschnittsuntersuchungen der Versuch unternommen wurde, diesem Mangel durch Kausalanalysen zu begegnen (Gentile, et al., 2004; Mößle, et al., 2007).

Um den Zusammenhang von Mediennutzung und Schulleistung zu erklären, wurde eine Reihe von Wirkhypothesen vertreten, die sowohl auf leistungsförderliche als auch auf leistungsmindernde Aspekte der Mediennutzung rekurrieren (vgl. Kapitel 1.2.3). Minderungshypothesen schließen dabei verhaltensbezogene (Zeitverdrängung), affektive (Aggressionshypothese) und kognitive Wirkpfade (Passivitäts-, Lesebeeinträchtigungs-, Konzentrations-Defizit-, Gedächtnis-Defizit-Hypothese) mit ein. Obgleich Stimulierungshypothesen zumindest für die Unterhaltungsmediennutzung und insbesondere die Nutzung gewalthaltiger Medien inzwischen als vernachlässigbar gelten müssen, haben die vielfältig postulierten Minderungshypothesen - mit Ausnahme der Zeitverdrängungshypothese und in Ansätzen der Konzentrations-Defizit-Hypothese - noch keine überzeugende empirische Bewährung erfahren. Hierbei ist zudem zu berücksichtigen, dass sowohl die Ableitung der Wirkhypothesen als auch ihre empirische Überprüfung bislang überwiegend im Hinblick auf das Fernsehen erfolgt ist. Gerade die Frage, wie sich Computerspiele auf kognitive und affektive Verarbeitungsvorgänge auswirken und 
in der Folge auch Veränderungen schulischer Leistungsparameter begründen könnten, ist bislang kaum erforscht worden.

Bei Betrachtung der Forschungslage zu den kognitiven Wirkungen von Computerspielen im Allgemeinen (vgl. Kapitel 1.3.2) und den emotionalen Wirkungen gewalthaltiger Computerspiele im Besonderen (vgl. Kapitel 1.3.3) kann im übertragenen Sinne der Eindruck einer Brücke entstehen, deren Aufbau von zwei Ufern beginnend aufgenommen wurde, deren Teilsegmente jedoch bislang nicht miteinander verbunden wurden: Das erste Brückenstück ist die Forschung zu den Auswirkungen von Computerspielen auf die kognitive Leistungsfähigkeit. Hier zeigt sich, dass bestimmte Computerspiele ein kompetenzförderliches Potential aufweisen, indem räumlich-visuelle Verarbeitungsleistungen sowie bestimmte Fertigkeiten trainiert werden. Das zweite Brückenstück ist der große Forschungszweig zu den Wirkungen von gewalthaltigen Spielen auf emotionale Erlebniszustände. Hier zeigen sich in Folge der Nutzung gewalthaltiger Spiele erhöhte physiologische Erregungsreaktionen und katecholamine Stressreaktionen sowie ein differenziertes Spektrum von emotionalen Erlebniszuständen, welches die erregungssteigernde Wirkung der Spiele plausibel erklären kann.

Besonderer Forschungsbedarf kann hinsichtlich des mittleren Brückenstücks angemeldet werden. So wurde bislang kaum untersucht, inwieweit affektive und kognitive Wirkungen gewalthaltiger Spiele ineinandergreifen und sich wechselseitig bedingen können. Unter Rückgriff auf die Forschung zum Zusammenhang von Mediennutzung und Schulleistung und den daraus abgeleiteten Wirkhypothesen stellt sich insbesondere die Frage, ob konkurrierende Informationsverarbeitungsprozesse (etwa in der Schule) durch das affektive Wirkspektrum beeinflusst werden können. In Kapitel 1.4 dieser Arbeit konnte gezeigt werden, dass aversive emotionale Reaktionen und Stressreaktionen eng miteinander verknüpft sind und Einfluss auf wesentliche Phasen der Informationsverarbeitung nehmen können:

1. Die Encodierung kann durch emotionale Prä-Learning-Reize beeinflusst werden. Abhängig von der Stärke der Emotionen kann es zu einer Steigerung oder Verminderung der Encodierungsleistung kommen.

2. Die Konsolidierung kurzfristig zuvor gelernter und damit noch störanfälliger Informationen kann durch emotionale Post-Learning-Reize beeinflusst werden.

Abhängig von der Stärke der Stressreaktion kann es zu einer Steigerung oder Verminderung der Konsolidierungsleistung kommen.

Damit stellt sich in Anknüpfung an die dargelegten kognitiven Interferenzhypothesen (vgl. Kapitel 1.2.3) die Frage, ob ähnliche Prozesse auch bei der Nutzung gewalthaltiger Medien wirksam werden können. 


\subsubsection{Fragestellungen}

Diese Arbeit konzentriert sich auf Computerspiele als vergleichsweise neues interaktives Unterhaltungsmedium. Willoughby (2008, S. 195) betont, dass gerade aufgrund des Anstiegs der Nutzung interaktiver Unterhaltungsmedien der Erforschung der kognitiven Auswirkungen dieses Mediums eine besondere Bedeutung zukommt. Gleichzeitig kann festgehalten werden, dass gerade mit der zunehmenden Übertragung klassischer Erklärungshypothesen zum Zusammenhang von Fernsehnutzung und Schulleistung auf Computerspiele ein dringender Bedarf an zusätzlicher Wirkungsforschung besteht, um die Relevanz der Hypothesen für diesen neuen Gegenstand abschätzen zu können.

Mittels einer experimentellen Untersuchung sollen Primärwirkungen interaktiver Medien auf den für schulisches Lernen in besonderer Weise relevanten Erwerb semantischer Wissensbestände überprüft werden. Dabei konzentriert sich diese Arbeit auf zwei wesentliche Gedächtnisfunktionen: Die Arbeitsgedächtnisleistung, die eine hohe Beanspruchung exekutiver Strukturen erfordert sowie die Konsolidierung bereits initial gelernter semantischer Wissensbestände. Die Reproduktionsleistung semantischer Wissensbestände wird dabei als Maß für die erfolgreiche Konsolidierung und Vernetzung der Informationen im Langzeitgedächtnis verstanden. ${ }^{55}$ Die folgenden Fragestellungen sollen beantwortet werden:

1. Welche Faktoren tragen dazu bei, dass bei der Rezeption gewalthaltiger Medien in besonderem Maße Distress erlebt wird?

2. Welchen Einfluss hat die Rezeption von gewalthaltigen Bildschirmmedien auf die Konsolidierung von Lerninhalten?

3. Welchen Einfluss hat die Rezeption von gewalthaltigen Bildschirmmedien auf die Arbeitsgedächtnisleistung?

4. Inwieweit weisen gewalthaltige Computerspiele und gewalthaltige Filme hinsichtlich des emotionalen und kognitiven Wirkpotentials eine differentielle Bedeutung auf?

55 Die Reproduktion gelernter Informationen stellt höhere kognitive Anforderungen als die reine Wiedererkennung gelernter Informationen. Da schulischer Lernerfolg sich in der Regel an der Fähigkeit entscheidet, gelernte Informationen in bestimmten Situationen (z. B. bei Klassenarbeiten) erfolgreich abrufen und im Rahmen von Problemlöseprozessen einsetzen zu können, wird diesem Maß der Vorzug gegeben. 


\subsubsection{Objektive Reizintensität und Distresserleben}

Es wird die Annahme zugrunde gelegt, dass Bildschirmmedienangebote Distress auslösen können (vgl. Kapitel 1.3.3). Dabei wird angenommen, dass Distress generell stärker bei der Rezeption gewalthaltiger Medien erlebt wird als bei der Rezeption gewaltneutraler Medien oder im Rahmen nicht-medialer Freizeitaktivitäten (vgl. Kapitel 1.3.3.1). Es wird ferner vermutet, dass die Nutzung interaktiver Mediengewalt insgesamt mit stärkerem Distress einhergeht als die Nutzung nicht-interaktiver Mediengewalt. Diese Annahme stützt sich auf die ausgeführten Erkenntnisse zur Häufigkeit von Gewaltereignissen in gewalthaltigen Computerspielen (vgl. Kapitel 1.1.2) und die damit verbundene erhöhte objektive Reizintensität von gewalthaltigen Computerspielen gegenüber gewalthaltigen Filmen vergleichbarer Genrekategorien (vgl. Kapitel 1.4.4.1). Weitere Unterschiede zwischen den Treatmentbedingungen werden in Hinblick auf objektive Merkmale des Mediums, die das Erleben von Distress kennzeichnen, nicht postuliert. Damit ergibt sich eine Haupteffekthypothese für einen Faktor A (Gewalt) und eine Interaktionseffekthypothese zwischen einem Faktor A (Gewalt) und einem Faktor B (Medium):

Hypothese A1: Die Rezeption gewalthaltiger Medien führt zu einem stärkeren Erleben von Distress als die Rezeption gewaltneutraler Medien.

Hypothese A2: Die Rezeption gewalthaltiger Computerspiele führt zu einem stärkeren Erleben von Distress als die Rezeption gewalthaltiger Filme.

\subsubsection{Subjektive Reizintensität und Distresserleben}

Für die Wirkannahmen dieser Arbeit ist das durch die mediale Gewalt vermittelte Erleben von Distress von zentraler Bedeutung. Ein Ausbleiben von Distress bei der Rezeption würde im Rahmen der hier postulierten Wirkzusammenhänge auch ein Ausbleiben kognitiver Beeinträchtigung erwarten lassen (vgl. Kapitel 1.4.3). Hierbei wird angenommen, dass konstituierende Merkmale auf Seiten der Rezipienten den Zusammenhang zwischen Mediengewaltrezeption und emotionalen Erlebniszuständen als subjektive Stressmoderatorvariablen moderieren und damit von Bedeutung dafür sein können, in welchem Maße Distress erlebt wird (vgl. Kapitel 1.4.4.2). In Abbildung 12 wird diese Zusammenhangsannahme veranschaulicht. 


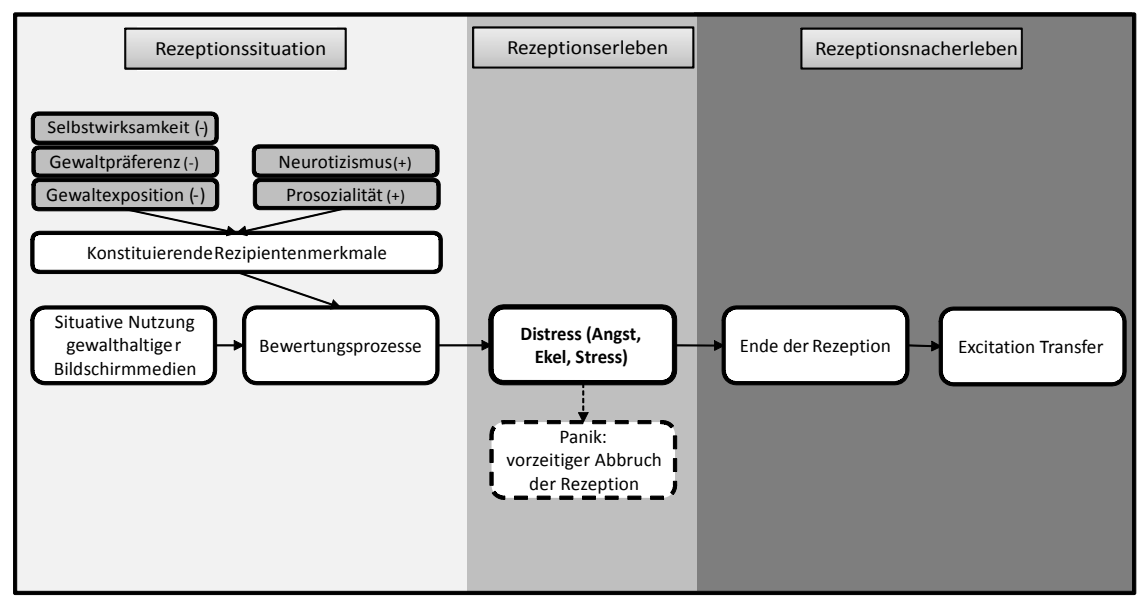

Abbildung 12. Angenommene Wirkpfade zwischen Gewaltmedienrezeption und dem Erleben von Distress

Unter Rückgriff auf die Diskussion moderierender Faktoren der subjektiven Reizintensität gewalthaltiger Computerspiele (vgl. Kapitel 1.4.4.2) wird angenommen, dass erhöhter Neurotizismus und erhöhte Prosozialität die Wahrscheinlichkeit dafür erhöhen, dass bei der Rezeption gewalthaltiger Bildschirmmedien Distress erlebt wird. Zudem wird angenommen, dass geringere Selbstwirksamkeitserwartung, geringere Gewaltpräferenz und geringere habituelle Gewaltmedienexposition die Wahrscheinlichkeit dafür erhöhen, dass bei der Rezeption gewalthaltiger Bildschirmmedien Distress erlebt wird. Es wird zudem unter Rückgriff auf die Ausführungen zu Angstreaktionen in Zusammenhang mit der Nutzung gewalthaltiger Medien (vgl. Kapitel 1.3.3.2.2) vermutet, dass weibliche Probanden in stärkerem Maße Distress erleben als männliche Probanden, dass dieser Geschlechterunterschied jedoch durch die oben genannten Variablen aufgeklärt werden kann.

Hypothese B1: Erhöhter Neurotizismus, erhöhte Prosozialität, geringere Selbstwirksamkeitserwartung, geringere Gewaltpräferenz und geringere habituelle Gewaltmedienexposition sagen Distresserleben bei der Rezeption gewalthaltiger Medien vorher.

Hypothese B2: Weibliche Geschlechtszugehörigkeit sagt Distresserleben bei der Rezeption gewalthaltiger Medien vorher. Das stärkere Erleben von Distress bei weiblichen Personen lässt sich dabei mittels der in Hypothese B1 berücksichtigten Prädiktoren aufklären. 


\subsubsection{Objektive Reizintensität und Kognition}

Allen kognitiven Wirkhypothesen dieser Arbeit liegt die Annahme zugrunde, dass gewalthaltige Medien Distress auslösen können (vgl. Kapitel 1.3.3.1, Kapitel 1.4.4). Die kognitiven Wirkhypothesen stützen sich dabei zum einen auf zwei populäre kognitive Interferenzhypothesen, die Konzentrations-Defizit-Hypothese und die Gedächtnis-Defizit-Hypothese (vgl. Kapitel 1.2.3), zum anderen auf Erkenntnisse zu den Auswirkungen akuter Stressreaktionen auf Gedächtnisfunktionen (vgl. Kapitel 1.4.3). Unter Rückgriff auf diese Ausführungen wird postuliert, dass Enkodierungs- und Konsolidierungsleistung unter dem Einfluss gewalthaltiger Medien schlechter ausfallen als unter dem Einfluss gewaltneutraler Medien.

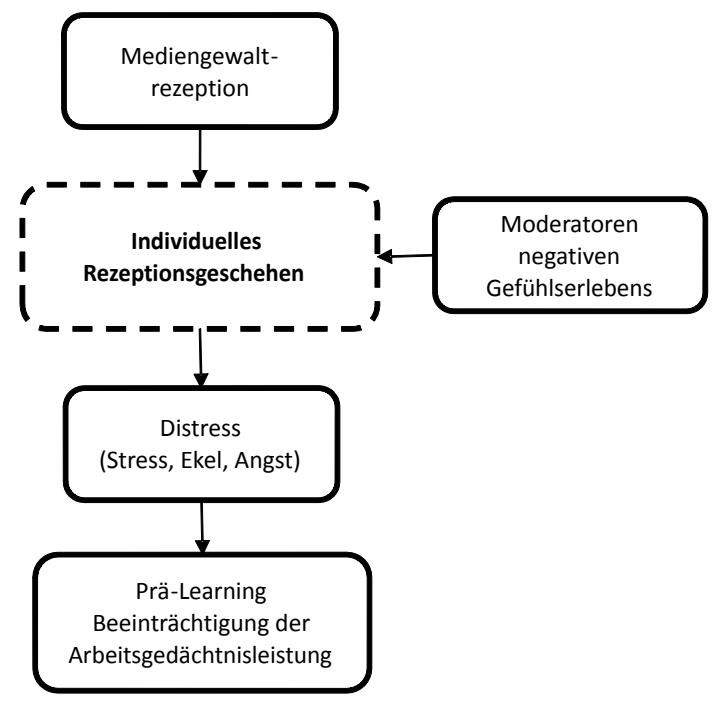

Abbildung 13. Vermuteter Einfluss von Mediengewaltrezeption auf die postrezeptive Arbeitsgedächtnisleistung

Hinsichtlich der Enkodierungsleistung wird angenommen, dass im Rahmen der Gewaltmedienrezeption erlebter Distress postrezeptiv zu einer Beeinträchtigung der Arbeitsgedächtnisleistung führt (vgl. Abbildung 13). Der Erregungsübertragungstheorie von Zillmann (2003) zufolge können rezeptionsbedingte emotionale Erregungszustände über die Rezeptionssituation hinaus bestehen bleiben (vgl. Kapitel 1.3.3.2.3). In Einklang mit dieser Theorie stehen erste Erkenntnisse zum über die Rezeptionssituation hinaus anhaltenden Erregungspotential gewalthaltiger 
Spiele (vgl. Kapitel 1.3.3.1) sowie retrospektive Befragungen zu Persistenz von Angstreaktionen in Folge der Nutzung gewalthaltiger Medien (vgl. Kapitel 1.3.3.2.2). Somit wird angenommen, dass Aufgaben, die in hohem Maße Arbeitsgedächtnisfunktionen binden, im Anschluss an die Nutzung gewalthaltiger Medien weniger effizient ausgeführt werden können. Es wird vermutet, dass diese Beeinträchtigung mittels eines Konzentrationsleistungstests, der in besonderer Weise das Arbeitsgedächtnis beansprucht, abgebildet werden kann. Hieraus wird die folgende Hypothese abgeleitet:

Hypothese C1: Die Rezeption gewalthaltiger Medien führt postrezeptiv zu einer geringeren Konzentrationsleistung als die Rezeption gewaltneutraler Medien.

Hinsichtlich der Konsolidierung wird angenommen, dass aufgrund des gewaltrezeptivbedingten Erlebens von Distress die Verarbeitung initial erlernter semantischer Informationen behindert wird (vgl. Abbildung 14). Es konnte gezeigt werden, dass explizite Gedächtnisfunktionen durch Stress in der Regel negativ beeinflusst werden (vgl. Kapitel 1.4.3). Dabei wird davon ausgegangen, dass gerade neu erworbene, emotional neutrale Wissensbestände noch instabil und daher anfällig für Post-Learning-Stress sind. Somit wird angenommen, dass die Konsolidierung von schulähnlichen Lerninhalten durch die Nutzung gewalthaltiger Medien beeinträchtigt werden kann. Hieraus wird die folgende Hypothese abgeleitet:

Hypothese C2: Die Rezeption gewalthaltiger Medien führt gegenüber der Nutzung gewaltneutraler Medien zu einer Beeinträchtigung der Konsolidierung expliziter räumlich-visueller und verbaler Gedächtnisinhalte. 


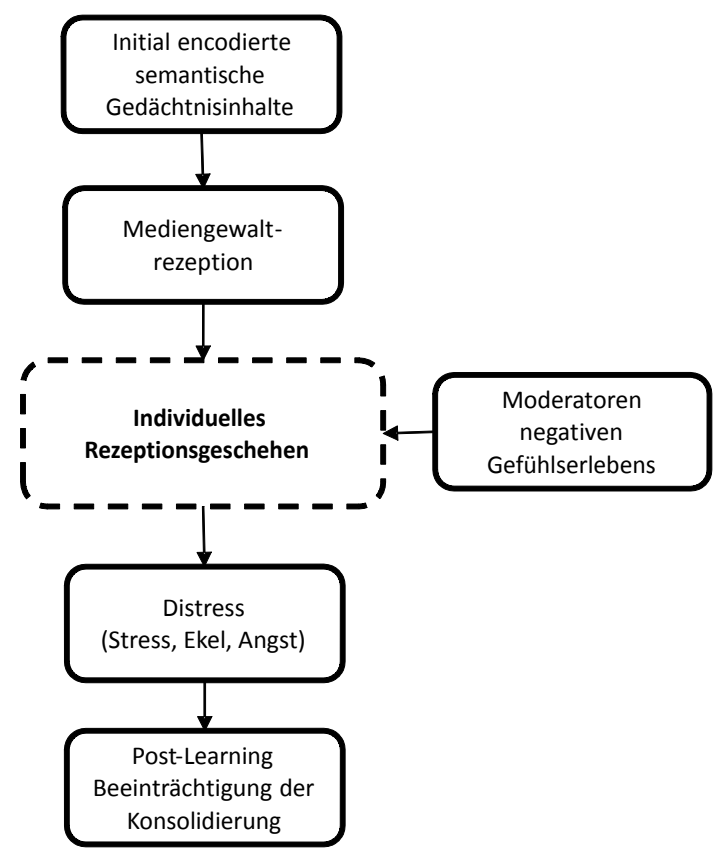

Abbildung 14. Vermuteter Einfluss von Mediengewaltrezeption auf die Konsolidierung semantischer Gedächtnisinhalte

Schließlich wird, da hinsichtlich gewalthaltiger Computerspiele ein objektiv höheres Erregungspotential postuliert wird als hinsichtlich gewalthaltiger Filme, angenommen, dass diese auch die betrachteten kognitiven Prozesse stärker beeinträchtigen. Hieraus wird die folgende Hypothese abgeleitet:

Hypothese C3: Die Rezeption gewalthaltiger Computerspiele führt hinsichtlich Konzentrationsleistung und Konsolidierung zu einer stärkeren Beeinträchtigung als die Rezeption gewalthaltiger Filme.

\subsubsection{Subjektive Reizintensität und Kognition}

Da Distress den vermuteten Mediator zwischen Gewaltmediennutzung und Kognition bildet wird angenommen, dass die selben Variablen, welche negative Gefühlsreaktionen bei der Nutzung gewalthaltiger Medien vorhersagen, auch den $\mathrm{Zu}$ sammenhang von Gewaltmediennutzung und Kognition moderieren. Es konnte gezeigt werden, dass sich die Wirkung von Stress auf explizite Gedächtnisfunktionen am besten im Rahmen einer umgekehrten U-Funktion beschreiben lässt, 
indem moderate Erhöhungen des Stresslevels keine negativen, sondern eher positive Wirkungen auf die Gedächtnisleistung erwarten lassen, während erhöhter Stress in der Regel beeinträchtigend wirkt (vgl. Kapitel 1.4.3). Zudem wurde ausgeführt, dass Menschen auf relative Stressoren interindividuell sehr unterschiedlich reagieren (vgl. Kapitel 1.4.2). Damit kann davon ausgegangen werden, dass subjektive Stressmoderatorvariablen in bedeutsamer Weise darüber entscheiden, ob und wenn ja in welcher Intensität die Rezeption gewalthaltiger Medien kognitive Beeinträchtigungen auslöst.

Vor diesem Hintergrund soll überprüft werden, ob der relevanteste aufgefundene Prädiktor von Distresserleben auch die Wirkungen von Gewalt auf kognitive Leistungen moderiert:

Hypothese D1: Der relevanteste Prädiktor von Distresserleben moderiert den Einfluss von Gewaltmedienrezeption auf die postrezeptive Konzentrationsleistung.

Hypothese D2: Der relevanteste Prädiktor von Distresserleben moderiert den Einfluss von Gewaltmedienrezeption auf die Konsolidierung räumlich-visueller und verbaler Gedächtnisinhalte. 


\section{Empirischer Teil}

\subsection{Methode}

Um sowohl die Nutzung medialer Stimuli als auch die Lern- und Leistungsergebnisse in ausreichender Weise standardisieren zu können und den Einfluss möglicher Störvariablen gering zu halten, wurde einem laborexperimentellen Zugang der Vorzug gegenüber einem feldexperimentellen Ansatz gegeben. Aufgrund der beabsichtigten Erfassung irreversibler Lerneffekte und der zeitlichen Beanspruchung der Versuchsteilnehmer durch die einzusetzende Testdiagnostik kam hinsichtlich der zu realisierenden Treatmentbedingungen nur ein Between-Subject-Design in Frage. Wie dargestellt wurde, lässt die bisherige Forschungslage den Schluss zu, dass sich unterschiedliche mediale Darbietungsformen und Inhalte in unterschiedlicher Weise auf kognitive Prozesse auswirken können. Daher berücksichtigt das Untersuchungsdesign sowohl einen Faktor A (Gewalt) als einen Faktor B (Medium). Der Faktor A (Gewalt) wurde zweifachgestuft variiert, indem gewalthaltige und gewaltneutrale Medien eingesetzt wurden. Der Faktor B (Medium) wurde zweifachgestuft variiert, indem als interaktives Medium Computerspiele und als nicht-interaktives Medium Filme eingesetzt wurden. Durch den Einbezug beider Faktoren können Interaktionen zwischen der Art des Mediums und dem dargeboten Inhalt ermittelt werden. Zusätzlich wurde eine fünfte nicht-mediale Explorationsbedingung realisiert, um veranschaulichen zu können, wie sich die vier hinsichtlich Gewalt und Interaktivität variierten Medienbedingungen in ihren Auswirkungen jeweils von einer nicht-medialen Freizeitbeschäftigung unterscheiden (vgl. Tabelle 8$)$.

Tabelle 8. Übersicht über das realisierte zweifaktorielle Untersuchungsdesign mit zusätzlicher nicht-medialer Explorationsbedingung

\begin{tabular}{ccccc}
\hline $\mathrm{A}_{1}$ & $\mathrm{~A}_{2}$ & $\mathrm{~A}_{1}$ & $\mathrm{~A}_{2}$ & \\
neutral & gewalthaltig & neutral & gewalthaltig & Nicht-mediale \\
$\mathrm{B}_{1}$ & $\mathrm{~B}_{1}$ & $\mathrm{~B}_{2}$ & $\mathrm{~B}_{2}$ & Explorations- \\
Spiele & Spiele & Filme & Filme & bedingung \\
X11 & $\mathbf{X 2 1}$ & $\mathbf{X 1 2}$ & $\mathbf{X 2 2}$ & \\
\hline
\end{tabular}

